Schweizerisches Jahrbuch für Entwicklungspolitik

27-2 | 2008

Migration und Entwicklung : Eine Zweckallianz

\title{
Rückkehrhilfe der Schweiz : Bilanz und Perspektiven
}

Eric Kaser and Saskia Schenker

\section{(2) OpenEdition}

1 Journals

Electronic version

URL: http://journals.openedition.org/sjep/344

DOI: 10.4000/sjep.344

ISSN: 1663-9677

Publisher

Institut de hautes études internationales et du développement

Printed version

Date of publication: 1 décembre 2008

Number of pages: $207-220$

ISBN: 978-2-940415-08-3

ISSN: 1660-5926

\section{Electronic reference}

Eric Kaser und Saskia Schenker, «Rückkehrhilfe der Schweiz : Bilanz und Perspektiven »,

Schweizerisches Jahrbuch für Entwicklungspolitik [Online], 27-2 | 2008, Online erschienen am: 31 Mai

2010, abgerufen am 08 September 2020. URL : http://journals.openedition.org/sjep/344 ; DOI :

https://doi.org/10.4000/sjep.344 


\title{
Rückkehrhilfe der Schweiz: Bilanz und Perspektiven
}

\author{
Eric Kaser und Saskia Schenker*
}

\section{Einleitung}

Im Jahr 2007 hat die Rückkehrhilfe der Schweiz ihr zehnjähriges Jubiläum gefeiert. Das Instrument der Rückkehrhilfe hat sich bewährt und stetig weiterentwickelt. Ziel des vorliegenden Artikels ist es, die Entwicklung der Rückkehrhilfe von ihren Anfängen bis ins Jahr 2008 anhand eines historischen Abrisses darzustellen sowie die verschiedenen Instrumente zu erläutern und nach einer Bilanz über eine zehnjährige Tätigkeit im Bereich der Rückkehrhilfe die Zukunftsperspektiven der Rückkehrhilfe aufzuzeigen.

Wer kann heutzutage Rückkehrhilfe in der Schweiz erhalten? Rückkehrhilfe kann von allen Personen aus dem Asylbereich bei den Rückkehrberatungsstellen in den Kantonen, in den Empfangsstellen und im Flughafentransit beantragt werden. Auch anerkannte Flüchtlinge können Rückkehrhilfe erhalten, wenn sie in ihr Heimatland zurückkehren wollen. Von der Rückkehrhilfe ausgeschlossen sind Personen, die straffällig geworden sind, oder die sich während des Verfahrens bzw. danach missbräuchlich verhalten haben.

Bestimmte Personengruppen im Ausländerbereich haben ebenfalls Zugang zur Rückkehrhilfe. Es sind dies Opfer und Zeugen von Menschenhandel sowie Cabaret-TänzerInnen, die sich in der Schweiz in einer Ausbeutungssituation befinden.

Folgende Personenkategorien des Asylbereichs haben Zugang zur Rückkehrberatung: Asylsuchende mit hängigem Verfahren, abgewiesene Asylbewerber, Personen mit Nichteintretensentscheid, vorläufig Aufgenommene, Schutzbedürftige und anerkannte Flüchtlinge ${ }^{1}$.

\section{Historischer Abriss der Rückkehrhilfe der Schweiz}

\section{Erste Rückkehrhilfemassnahmen}

Bereits im Jahr 1959 eröffnete die Polizeiabteilung des Eidgenössischen Justizund Polizeidepartements (EJPD) ein Dossier zur Rückwanderung von Flüchtlingen. Für bedürftige südamerikanische Flüchtlinge wurden bis Mitte der 80er

* Eric Kaser, Leiter der Sektion Rückkehrhilfe, Bundesamt für Migration (BFM), Eidgenössisches Justiz- und Polizeidepartement (EJPD).

Saskia Schenker, Mitarbeiterin der Sektion Rückkehrhilfe, Bundesamt für Migration (BFM), Eidgenössisches Justiz- und Polizeidepartement (EJPD).

1 BFM, „Rückkehrhilfe“, <http://www.bfm.admin.ch/bfm/de/home/themen/rueckkehr/rueckkehrfoerderung. html>. 
Jahre von Fall zu Fall Flugtickets oder Transportkosten übernommen, bzw. Reisegelder ausgezahlt. Die konzeptionellen Anfänge der heutigen Rückkehrhilfe gehen auf das Jahr 1985 zurück. Vor dem Hintergrund entsprechender Anträge einiger Hilfswerke im Zuge einer verstärkten Rückkehrbewegung südamerikanischer Flüchtlinge und anlässlich der bevorstehenden Revision des Asylgesetzes regte das damalige Bundesamt für Polizeiwesen, Abteilung Flüchtlinge, die Schaffung einer interdepartementalen Arbeitsgruppe Rückkehrhilfe an (siehe Rahmentext zur Chronologie der Rückkehrhilfemassnahmen).

\section{Chronologie der Rückkehrhilfemassnahmen}

๑ 1959 : Eröffnung eines ersten Dossiers „Rückwanderung von Flüchtlingen nach ihrer ursprünglichen Heimat" durch die Polizeiabteilung des Eidgenössischen Justiz- und Polizeidepartements (EJPD) aufgrund eines Gesuchs um Mithilfe bei der Beschaffung von Papieren für zwei ungarische Flüchtlinge, die in ihre Heimat zurückkehren wollten.

๑ 70er Jahre bis Mitte der 80er Jahre: Ausstellung von Flugtickets oder Übernahme von Transportkosten plus Reisegeld bei Einzelanfragen von überwiegend südamerikanischen Flüchtlingen.

- 1985 : Erste interdepartementale Arbeitsgruppe Rückkehrhilfe (Bundesamt für Polizeiwesen, Abteilung Flüchtlinge, und Departement für auswärtige Angelegenheiten, EDA).

ـ 1986 : Interdepartementale Arbeitsgruppen Chile, Zaire (heutige Demokratische Republik Kongo) und Türkei. Das revidierte Asylgesetz enthält eine Bestimmung zur Gewährung von Rückkehrhilfe.

- 1987 : Umsetzung des ersten Rückkehrhilfeprogramms („Programm für Rückkehrhilfe und berufliche Wiedereingliederung von Chilenen") und erste Rückkehrberatungsstellen in der Schweiz. In Chile wurde das Programm durch die heutige Internationale Organisation für Migration (IOM), damals Intergovernmental Committee for Migration (ICM), umgesetzt.

ـ 1993 : Erstellung des ersten schweizerischen Rückkehrhilfekonzepts durch das damalige Bundesamt für Flüchtlinge (BFF) und Einsatz eines Projektleiters Rückkehrhilfe im BFF.

- 1994 : Eröffnung des IOM-Büros in Bern.

ـ 1996 : Lancierung des Pilotprogramms Bosnien und Herzegowina.

๑ 15. Januar 1997 : Einführung der allen Asylsuchenden zugänglichen Rückkehrhilfe durch Versand von Weisungen über die Rückkehrberatungsstellen und über die individuelle Rückkehrhilfe. Aufbau des Rückkehrberatungsnetzes. Lancierung des Rückkehrhilfeprogramms Bosnien und Herzegowina (19971999).

- 1998 : Aufnahme der Rückkehrhilfe in die Bestimmungen des Asylgesetzes.

๑ 1999 : Schaffung der Sektion Rückkehrhilfe im BFF. Rückkehrhilfeprogramm Kosovo (1999-2001).

\section{Entwicklung der Länderprogramme}

Die breite Förderung der freiwilligen Rückkehr wurde auf Bundesebene zum ersten Mal mit dem Rückkehrhilfeprogramm Bosnien und Herzegowina umgesetzt. Das Programm hat in einem Zeitraum von zweieinhalb Jahren die freiwillige Rückkehr von rund 10000 Personen in ihre Heimat ermöglicht. Auf Grund der gemachten Erfahrungen wurden im damaligen Bundesamt für Flüchtlinge (BFF) die Strukturen ergänzt und eine neue Sektion Rückkehrhilfe geschaffen. Gleichzeitig wurde das bis heute bestehende Netz der Rückkehrberatungsstellen in den Kantonen aufgebaut. Der Gedanke, dass durch geeignete Anreize, gezielte Information und adäquate Beratung die Rückkehr von Flüchtlingen und Asylsuchenden gefördert werden könne, hatte sich endgültig durchgesetzt. 
Nach der Einstellung der Kampfhandlungen im Kosovo und der Stationierung friedenserhaltender Truppen wurde die vorläufige kollektive Aufnahme von Kriegsvertriebenen in der Schweiz per Bundesratsbeschluss vom 16. August 1999 aufgehoben und eine Ausreisefrist angesetzt. Das BFF initiierte darauf zusammen mit der Direktion für Entwicklung und Zusammenarbeit (DEZA) des Eidgenössischen Departements für auswärtige Angelegenheiten (EDA) und der Internationalen Organisation für Migration (IOM) das Rückkehrhilfeprogramm Kosovo. Dieses Programm war mit Gesamtkosten von 184 Millionen Franken ein Projekt von einmaliger Grössenordnung. In den Kosten mit eingeschlossen war neben den Rückkehrhilfeleistungen ein Beitrag zum internationalen Wiederaufbau des Kosovo, der sich an die gesamte Bevölkerung und nicht spezifisch an Rückkehrer richtete. Die Ausarbeitung und die Implementierung dieser Strukturhilfemassnahmen wurden von der DEZA übernommen.

Obwohl die beiden oben erwähnten Rückkehrhilfeprogramme im westlichen Balkan sowohl aufgrund der hohen Rückkehrzahlen als auch wegen der Gesamtkosten eine Ausnahme darstellten und alle zukünftigen Projekte nicht mehr diesem Umfang entsprachen, konnten die guten Erfahrungen aus beiden Programmen auf die darauf folgenden Programme übertragen werden.

Die Länderprogramme werden von der Sektion Rückkehrhilfe des heutigen Bundesamts für Migration (BFM) zusammen mit der DEZA und der IOM Bern erarbeitet. Dabei sollen Rückkehrhilfemassnahmen umgesetzt werden, die speziell an die Zielgruppen und an die Situation der Herkunftsländer angepasst sind. Länderprogramme beinhalten eine Rückkehrhilfekomponente mit Finanzierung eines Partners vor Ort und eine Strukturhilfekomponente, die durch die DEZA umgesetzt wird. Die Rückkehrhilfekomponente enthält für den Rückkehrer zusätzliche Leistungen im Vergleich zur individuellen Rückkehrhilfe. Folgende Programme wurden seit den ersten Balkan-Programmen umgesetzt (siehe Rahmentext).

\section{Länderprogramme}

口 2000 : Programm Sri Lanka (2000-2004).

口 2001 : Programm Äthiopien (2001-2002), Bundesrepublik Jugoslawien (2001-2003), Nordirak (20012003), Somalia (2001-2002).

๑ 2002 : Programm Angola (2002-2007), Iran (2002-2003), Mazedonien (2002-2003), Minoritäten Kosovo (2002-2003)

口 2003 : Programm Balkan für vulnerable (verletzliche) Personen (2003-2006), Irak (seit 2003), Türkei (2003-2005).

๑ 2004 : Programm Armenien (2004-2008), Demokratische Republik Kongo (2004-2006).

๑ 2005 : Programm Nigeria (seit 2005), Westafrika (seit 2005).

๑ 2006 : Programm Äthiopien (2006-2008), Afghanistan (2006-2008), Georgien (seit 2006), Maghreb (seit 2006)

口 2007 : Beitrag der Interdepartementalen Leitungsgruppe Rückkehrhilfe (ILR) an die Migrationspartnerschaften Westbalkan und Rückkehrhilfeprogramm Balkan für vulnerable Personen und Minderheiten Kosovo (2007-2009). 
Artikel 93 des Asylgesetzes, ,Rückkehrhilfe und Prävention irregulärer Migration“, bildet die gesetzliche Grundlage für die Rückkehrhilfe der Schweiz. In der Asylverordnung 2, Artikel. 62-78, sind der Zweck, die Begünstigten und die Ausschlussgründe festgehalten.

Nachstehend werden folgende Instrumente der Schweizer Rückkehrhilfe präsentiert: Individuelle Rückkehrhilfe, Rückkehrberatungsstellen, Rückkehrhilfe ab Empfangs- und Verfahrenszentren, Pilotprojekt AuG, Strukturhilfeprojekte und Prävention irregulärer Migration.

\section{Individuelle Rückkehrhilfe}

Neben den Länderprogrammen bildet die individuelle Rückkehrhilfe (IHI) das Grundlagenangebot der Rückkehrhilfe, das für jedes Rückkehrland besteht. Die Leistungen der individuellen Rückkehrhilfe wurden in den letzten Jahren ausgebaut und entsprechen heute in Umfang und Höhe der Rückkehrhilfe in einem Länderprogramm. Alle freiwilligen RückkehrerInnen aus dem Asylbereich, unabhängig von ihrem Herkunftsland, haben Anrecht auf eine unverbindliche Beratung in der Rückkehrberatungsstelle des Aufenthaltskantons, auf die Organisation der Heimreise und die Entschädigung der Reisekosten. Zusätzlich gewährt die Rückkehrhilfe, bei einem Mindestaufenthalt von drei Monaten in der Schweiz, eine Starthilfe von 1000 Franken für Erwachsene und von 500 Franken für Minderjährige. Die Wiedereingliederungshilfe (materielle Zusatzhilfe) beträgt pro Fall maximal 3000 Franken, und es kann zwischen Beruf und Ausbildung, Wohnraum, Transport von Material oder Betreuung durch Familie (Cash for Shelter, Cash for Care) ausgewählt werden. Zusätzlich wird bei Vorliegen eines Arztzeugnisses medizinische Unterstützung für die erste Zeit nach der Rückkehr angeboten.

Für die Auszahlung der Rückkehrhilfe, die Unterstützung im Rahmen des beruflichen Projekts und die Hilfeleistungen bei der Ermittlung sozialer oder medizinischer Strukturen werden die Dienstleistungen von IOM-Missionen, diplomatischen Vertretungen der Schweiz oder von Nichtregierungsorganisationen als Partner vor Ort in Anspruch genommen.

Die Rückkehrhilfe kann aber lediglich einen Beitrag zur Erleichterung der Rückkehr und eine Starthilfe für die Reintegration vor Ort leisten. Die Begleitung durch die IOM dauert in der Regel sechs Monate bis ein Jahr. Zusätzliche Unterstützung oder eine Garantie zu einer erfolgreichen Wiedereingliederung kann die Rückkehrhilfe nicht übernehmen.

Mit Inkrafttreten des neuen Asylgesetzes im Jahr 2008 bleiben die einzigen Ausschlussgründe für Rückkehrhilfe, gemäss Asylverordnung 2 Artikel 64 des Asylgesetzes, die Straffälligkeit, die Verletzung der Mitwirkungspflicht und das Vorhandensein von genügend finanziellen Eigenmitteln. Zudem wurde die seit Mai 2007 geltende Praxis des Ausschlusses von Staatsangehörigen aus EU/EFTA-Staaten sowie aus traditionellen Auswanderungsländern (USA, Kanada, Australien) ins ordentliche Recht aufgenommen (Asylverordnung 2, Art. 76). Jede Person kann nur einmal Rückkehrhilfe erhalten. Stellt eine freiwillig 
zurückgekehrte Person ein weiteres Asylgesuch in der Schweiz, ist sie zur Rückzahlung der erhaltenen Rückkehrhilfe verpflichtet.

\section{Grafik 1: Wer braucht was?}

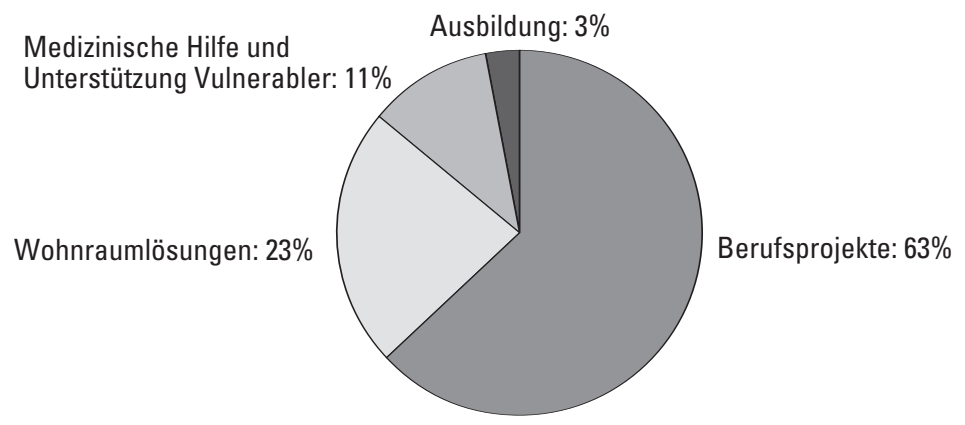

Quelle: Sektion Rückkehrhilfe, BFM, 2007.

\section{Rückkehrberatungsstellen (RKB)}

Die Rückkehrberatungsstellen (RKB) werden von den Kantonen eingesetzt und betrieben und vom Bundesamt für Migration (BFM) finanziert. Ihre Aufgabe ist es, berechtigten Personen bei der Ausarbeitung der Rückkehrperspektiven behilflich zu sein. Dazu gehören die Information an potenziell freiwillige Rückkehrer und die Aufrechterhaltung eines Dialogs im Umfeld der jeweiligen Zielgruppe sowie die Vernetzung von kantonalen und kommunalen Behörden. Durch individuelle Behandlung der einzelnen Fälle (Case Management) betreuen die Rückkehrberatungsstellen Rückkehrer von der ersten Beratung bis zur Ausreise. Die Vernetzung mit der Sektion Rückkehrhilfe des BFM und mit IOM Bern ist gewährleistet.

\section{Rückkehrhilfe ab Empfangs- und Verfahrenszentren (REZ)}

Im Jahr 2005 wurde das Projekt „Rückkehrhilfe ab Empfangs- und Verfahrenszentren" (REZ) eingeführt. Im Rahmen dieses Projekts wird den betroffenen Personen ab der Einreise in die Schweiz in den vier Empfangs- und Verfahrenszentren und im Transitzentrum des BFM eine begrenzte Rückkehrhilfe (Rückkehrberatung plus eine Pauschale von 500 Franken) gewährt. Umgesetzt wird dieses Projekt vom IOM-Büro in Bern.

\section{Pilotprojekt AuG}

Mit Inkrafttreten von Artikel 60 des Ausländergesetzes (AuG) am 1. Januar 2008 kann erstmals die selbständige und pflichtgemässe Ausreise von besonders bedürftigen AusländerInnen unterstützt werden. So wird bestimmten Personengruppen aus dem Ausländerbereich durch ein Pilotprojekt AuG der Zugang zur Rückkehrhilfe ermöglicht. Dieses zweijährige Pilotprojekt richtet sich zum einen an Opfer und Zeugen von Menschenhandel, zum anderen an Personen, die im Zusammenhang mit ihrer Erwerbstätigkeit der Gefahr der Ausbeutung 
besonders ausgesetzt sind. Hierbei handelt es sich um das erste Projekt, das themenspezifisch und somit länderübergreifend umgesetzt wird.

\section{Strukturhilfe und Prävention irregulärer Migration (PiM)}

Strukturhilfe kann in jedem Land umgesetzt werden, für dessen Staatsangehörige das BFM Rückkehrhilfe leistet. Die Ziele der Strukturhilfe sind:

$\square$ Die Disparitäten zwischen Rückkehrern und vor Ort Verbliebenen dadurch zu verringern, dass Strukturhilfeprojekte auch den vor Ort Verbliebenen zugute kommen.

๖ Einen Anreiz für den Herkunftsstaat zur Rückübernahme und zu eigenem Engagement für Reintegrationsmassnahmen zu schaffen.

• Einen Beitrag zur Entwicklung von Migrationsmanagement-Kapazitäten des Herkunftslandes zu leisten.

๖ Einen Beitrag zur Entwicklung von Migrationspartnerschaftsbeziehungen und als Unterstützung für den Migrationsdialog zu leisten.

Artikel 72, Absatz.2 der Asylverordnung 2 über Finanzierungsfragen hält fest, dass die DEZA die Auslandsprogramme des BFM umsetzt. Die aus dem Kredit für Rückkehrhilfe des BFM finanzierte Strukturhilfe ist für die DEZA als zusätzlicher Auftrag anzusehen. Neben den DEZA-Programmen, die sich aus dem Gesetz über die Entwicklungszusammenarbeit und die humanitäre Hilfe ergeben, hat die DEZA auch andere Aufgaben, die sich aus der Zusammenarbeit mit externen Partnern ableiten. Diese „Drittgeschäfte“ stehen im Einklang mit den Grundprinzipien der DEZA, setzen aber andere Schwerpunkte. So werden die vom BFM finanzierten Strukturhilfeprojekte den DEZA-Kriterien für Strukturhilfe unterstellt und die Projekte dementsprechend geprüft und ausgeschrieben. Das BFM greift auf die Expertise der DEZA zurück, ohne dass die Entwicklungszusammenarbeit und insbesondere die humanitäre Hilfe für die Interessen des BFM instrumentalisiert werden.

Gemäss dem am 1. Januar 2008 in Kraft getretenen neuen Artikel 93 des Asylgesetzes gilt, dass Programme im Ausland auch das Ziel verfolgen können, einen Beitrag zur Prävention irregulärer Migration (PiM) zu leisten. Anfang des Jahres 2008 wurde deshalb von der Interdepartementalen Leitungsgruppe Rückkehrhilfe (ILR) ein „Projektteam zur Prävention irregulärer Migration“ (PT PiM) eingesetzt. Im Rahmen dieses Projektteams werden in Schwerpunktländern Projekte zur Verhütung irregulärer Migration geplant und umgesetzt. Diese Projekte werden nach Genehmigung durch die ILR vom BFM finanziert. Projekte im Rahmen des Mandats des Projektteams zur Prävention irregulärer Migration verfolgen das Hauptziel, einen Beitrag auf operationeller Ebene zur kurzfristigen Minderung irregulärer Migration zu leisten. Konkret fallen in die Kategorie des Mandats Informations- und Aufklärungskampagnen für potenzielle Migranten und die Unterstützung für irreguläre Migranten bei der Rückkehr aus einem Transitland (so genannte „Stranded Migrants"). Im Gegensatz dazu schaffen Strukturhilfeprojekte Alternativen zur irregulären Migration. Die PiM-Schwerpunktländer variieren je nach migrationspolitischen Entwicklungen. 


\section{Interdepartementale Leitungsgruppe Rückkehrhilfe (ILR)}

Die Interdepartementale Leitungsgruppe Rückkehrhilfe (ILR) ist das Organ, das über die Durchführung von Länderprogrammen und Strukturhilfeprojekten im Bereich der Rückkehrhilfe entscheidet. Sie koordiniert die entsprechenden Aktionen, prüft das Synergiepotenzial von Massnahmen der DEZA und der Sektion Rückkehrhilfe des BFM sowie weiterer Stellen und vernetzt die Massnahmen mit dem Ausschuss der Interdepartementalen Arbeitsgruppe Migration (IAM-Ausschuss). Die ILR wurde 1999 institutionalisiert, nachdem die im Bosnienprogramm seit 1996 bestehende Zusammenarbeit des damaligen BFF, der DEZA und der IOM als erfolgreich beurteilt worden war. Die Leitung des Koordinationsbüros der IOM Bern, die für Migration und Entwicklung beauftragte Person der DEZA sowie die Vertretung der Politischen Direktion (PA IV) des EDA nehmen als ständige BeraterInnen in der ILR Einsitz ${ }^{2}$.

Mit der Institutionalisierung der ILR zur departementsübergreifenden Zusammenarbeit im Bereich der Rückkehrhilfe wurde ein Instrument geschaffen, das den Informationsaustausch und die Kohärenz bei der Arbeit der Rückkehrhilfe stärkt und eine Vernetzung der Bereiche Migration und Entwicklung ermöglicht.

\section{Beitrag der ILR zu den Migrationspartnerschaften Schweiz-Westbalkan 2007-2009}

Ein Beispiel zur Koordinationsfunktion der ILR bietet das Strategiepapier „Beitrag der ILR zu den Migrationspartnerschaften Schweiz-Westbalkan 20072009, Kosovo, Bosnien und Herzegowina, Serbien“3.

Die vier Ziele des ILR-Beitrags sind:

$\checkmark$ Beitrag zur Entwicklung von Migrationspartnerschaften der Schweiz mit Bosnien-Herzegowina, Serbien und dem Kosovo.

$\checkmark$ Förderung der freiwilligen Rückkehr insbesondere von Minderheitenangehörigen und vulnerablen (verletzlichen) Personen.

- Stärkung der Migrationsmanagement-Kapazitäten der beteiligten Staaten.

ـ Prävention irregulärer Migration durch soziale- und wirtschaftliche Strukturhilfe.

Die Rückkehrhilfekomponente wurde speziell auf vulnerable Personen und Minderheiten des Kosovos ausgerichtet. Die Unterstützung wird auf die individuellen Bedürfnisse der zurückkehrenden Personen abgestimmt, nachdem Abklärungen vor Ort vorgenommen wurden. Somit wird eine umfassende, auf die einzelnen RückkehrerInnen abgestimmte Rückkehrhilfe geleistet, was für die gewählte Zielgruppe notwendig ist.

ILR, Mandat, Bern, BFM; DEZA, 2005.

3 ILR, Beitrag der ILR zu Migrationspartnerschaften Schweiz-Westbalkan: Strategiepapier 2007-2009 Kosovo, Bosnien und Herzegowina, Serbien, Bern, BFM; DEZA, 2007. (Für Details zu den Migrationspartnerschaften siehe entsprechendes Kapitel.) 
Für die Strukturhilfe wurden drei thematische Projektlinien bestimmt:

I. Kapazitätenbildung für Migrationsmanagement;

II. Soziale und wirtschaftliche Entwicklung;

III. Reintegrationshilfe für Zurückgekehrte und Migrations-Informationsdienste.

Die Themenschwerpunkte werden im Rahmen des bilateralen Migrationsdialogs mit dem jeweiligen Land identifiziert und vereinbart. Die DEZA und ihre Kooperationsbüros vor Ort sind verantwortlich für die Projektkonzipierung und die Vorbereitung der Projektanträge zuhanden der ILR, die zuvor im Projektteam zusammen mit der Sektion Rückkehrhilfe des BFM besprochen werden. Die ILR entscheidet über die Finanzierung der Projekte, welche wiederum durch die DEZA umgesetzt werden. Das Budget von insgesamt 12 Millionen Franken für die Strukturhilfeprojekte wird vom BFM bereitgestellt. Zusätzlich beteiligt sich das Fürstentum Liechtenstein mit einem Beitrag von 1,5 Millionen Franken. An Projekte schweizerischer Hilfswerke, die bezüglich Zielsetzung, Zielgruppen, thematischer und geografischer Konzentration mit der ILRStrategie „Migrationspartnerschaften Westbalkan 2007-2009“ (MPWB 07-09) übereinstimmen, können im Rahmen der Strukturhilfe Finanzierungsbeiträge (bis maximal 50\%) geleistet werden. Die Hilfswerke werden somit in den Dialog rund um den Beitrag der ILR zu den Migrationspartnerschaften SchweizWestbalkan einbezogen.

\section{Bilanz über zehn Jahre Rückkehrhilfe}

\section{Bilanz betreffend die Rückkehrhilfekomponente}

\section{$\square$ Anpassung an neue Voraussetzungen}

Die Ausrichtung der Rückkehrhilfe hat sich von der anfänglichen blossen Auszahlung von Geldpauschalen zu einer auf die individuellen Bedürfnisse der RückkehrerInnen abgestimmten Wiedereingliederungsmassnahme hin entwickelt. Diese Orientierung erfolgte nicht nur in den spezifischen Länderprogrammen, da auch die individuelle Rückkehrhilfe, die für jedes einzelne Rückkehrland angeboten wird, kontinuierlich neuen Voraussetzungen angepasst wurde. Nach der Massenrückkehr in den ersten Balkanprogrammen und dem Rückgang der Anzahl von Personen, die mit Rückkehrhilfe ausreisen, haben die aufwändigen Rückkehrhilfefälle zugenommen - das heisst, Fälle von Personen, die nach vielen Jahren Aufenthalt in der Schweiz freiwillig in ihre Heimat zurückkehren wollen, die krank sind oder in Kliniken leben, allein erziehende Frauen, Witwen, Rentner, Familien, Personen mit Kriegstraumata oder Menschen, die besonders weite und schwierige Wege zurückgelegt haben. All diese freiwilligen RückkehrerInnen benötigen umfangreiche Abklärungen und Unterstützung, bevor sie die Rückreise in ihr Heimatland antreten können. Deshalb entsprechen die Rückkehrhilfeleistungen der individuellen Rückkehrhilfe heute in Höhe und Umfang weitgehend jenen in den Länderprogrammen. Dadurch wird dem Aspekt der Rückkehrmotivation besser Rechnung getragen, und die Rückkehrhilfekomponente in den Ländern, in denen ein Programm angeboten wird, unterscheidet sich nicht mehr signifikant von der individuellen Rückkehrhilfe. 


\section{$\square$ Rückkehrzahlen}

Insgesamt erhielten seit 1997 rund 65000 Personen Rückkehrhilfeleistungen der Schweiz, in den letzten fünf Jahren waren es durchschnittlich 1500 Personen pro Jahr. Der Balkan als ursprüngliche Schwerpunktregion der freiwilligen Rückkehr bleibt weiterhin wichtig. Im Rückkehrhilfeprogramm Westbalkan ist immer noch eine beachtliche Anzahl freiwilliger RückkehrerInnen zu verzeichnen. Im Durchschnitt sind dies für das Rückkehrhilfeprogramm Westbalkan (Bosnien und Herzegowina, Serbien, Kosovo, Montenegro) um die 50 Personen im Jahr, für Mazedonien ca. 30 Personen pro Jahr. In den letzten Jahren verlagerte sich die freiwillige Rückkehr aber schwerpunktmässig vermehrt auf die Länder der ehemaligen Sowjetunion und auf Afrika. Im Länderprogramm Georgien hält sich die Anzahl der freiwilligen RückkehrerInnen seit Beginn des Programms 2006 im Durchschnitt bei etwas über 30 Personen pro Jahr. Auch Russland und die Ukraine zählen bei der individuellen Rückkehrhilfe zu jenen Ländern, in die überdurchschnittlich viele Personen mit Rückkehrhilfe zurückkehren. Die Türkei verzeichnete in den Jahren 2006 und 2007 mehr als 60 freiwillig zurückkehrende Personen aus der Schweiz. In Afrika liegen die Schwerpunkte bei den Länderprogrammen Nigeria, Westafrika und Maghreb. Das Angola-Programm lief Ende 2007 aus. Bei der individuellen Rückkehrhilfe gilt Kamerun ebenfalls als Land mit einer überdurchschnittlichen Anzahl freiwilliger RückkehrerInnen.

Auch politische Entwicklungen beeinflussen die Zahlen der freiwilligen Rückkehr: So sind in den Jahren 2004-2006 im Rahmen des Länderprogramms Irak jeweils über 110 Personen pro Jahr freiwillig in den Irak zurückgekehrt.

\section{$\square$ Evaluation am Beispiel des Rückkehrhilfeprogramms Balkan für vulnerable Personen (2003-2006)}

Das Rückkehrhilfeprogramm Balkan für vulnerable Personen (2003-2006) orientierte sich erstmals an den individuellen Bedürfnissen der Personen, die aufgrund persönlicher Konstitution und Lage ausserordentliche Probleme bei der Wiedereingliederung im Heimatland hatten, bzw. deren Rückkehr unzumutbar oder nur schwer durchzuführen war, und die gleichzeitig für die Schweiz die grössten Herausforderungen hinsichtlich Rückkehr bzw. Integration bedeuteten.

Für das Programm meldeten sich vom 1. Juli 2003 bis zum 31. Dezember 2006 252 Personen an, davon kehrten 205 in ihre Heimat zurück, rund die Hälfte davon aus Bosnien-Herzegowina. Eine so genannte „Rück-Rückkehr“ gab es in drei Fällen.

Beim Familienprofil bildeten die 124 Personen der Alleinerziehenden mit ihren Kindern und die Familien die grösste Gruppe (zusammen 59\%). Alleinstehende machten 24\%, Ehepaare 16\% aus. Zwei Drittel der Ehepaare und knapp die Hälfte der Einzelpersonen waren über 60 Jahre alt. Dieses - gemessen am kleinen Anteil der über Sechzigjährigen - überdurchschnittliche Interesse an einer Rückkehr lässt sich in den meisten Fällen durch die fehlende Integration in der Schweiz, wie auch durch den Wunsch erklären, den Lebensabend in der Heimat zu verbringen. Zwei Personen kehrten totkrank in die Heimat zurück und starben kurz nach der Rückkehr. 
Bei knapp drei Viertel der Teilnehmer erfolgte die Rückkehr nach langer oder sehr langer Aufenthaltsdauer in der Schweiz. 42\% waren zwischen vier und sieben Jahren und 31\% länger als acht Jahre in der Schweiz. Sie reisten zu einem Zeitpunkt aus, zu dem die Integration in der Regel im Vordergrund steht, sofern sie nicht bereits erfolgt ist.

Im Auftrag der Interdepartementalen Leitungsgruppe Rückkehrhilfe (ILR) und des Projektteams Balkan verfasste IOM Bern im Herbst 2006 einen Monitoringbericht über sämtliche Personen, die zwischen dem 1. Juli 2003 und dem 31. August 2006 im Rahmen des Rückkehrprogramms für vulnerable Personen in den Balkan zurückgekehrt waren. Der Bericht sollte die Effektivität und die Nachhaltigkeit der Reintegrationsmassnahmen prüfen.

Die Ergebnisse dieser Einzelfallstudie können als Erfolg gewertet werden. Allein die Tatsache, dass rund 90\% der Rückkehrer immer noch an ihrem Rückkehrort wohnhaft sind, deutet darauf hin, dass sich ihre Situation nach der Rückkehr stabilisiert hat. Nur in zwei Fällen ist eine Weiterwanderung bekannt. Zwei Drittel gaben eine Unzufriedenheit über ihre Situation an. Hauptgründe dafür waren das Fehlen eines guten familiären Netzes (z.B. infolge von Scheidung, Tod von Angehörigen, Abwanderung eines Teils der Familie ins Ausland oder familieninternen Spannungen), eine schlechte gesundheitliche Verfassung oder der weiterhin bestehende Wunsch nach einer Migration nach Westeuropa. Dieser Umstand zeigt, dass in einigen Fällen Potenzial für strukturelle Verbesserungen vorhanden ist. Dabei muss gleichzeitig berücksichtigt werden, dass die Einflussnahme externer Institutionen in persönliche Lebensumstände begrenzt ist. In 37 Fällen erhielten Rückkehrer Unterstützung für die berufliche Reintegration und in 22 Fällen tragen diese Projekte weiterhin zum Einkommen der Rückkehrer bei. Dies entspricht drei Vierteln aller Projekte. In nahezu allen Fällen hat sich die Integration in die staatlichen Strukturen als problemlos erwiesen.

Die Unterstützungsleistungen, die im Rahmen des Programms angeboten werden, haben sich weitgehend bewährt. Ein Grossteil der Investitionen, welche mit Mitteln aus dem Programm getätigt wurden, ist immer noch im Besitz und Gebrauch der Rückkehrer. Somit kann durchaus von einer nachhaltigen Unterstützung gesprochen werden ${ }^{4}$.

Die Eidgenössische Finanzkontrolle (EFK) bestätigte in ihrem Schlussbericht zur Revision betreffend die Überprüfung der Abläufe und Prozesse im Bereich der Rückkehrhilfe für Asylsuchende den wirtschaftlichen Mitteleinsatz von Länderprogrammen und bezeichnete die dafür verantwortliche ILR ,,als Musterbeispiel einer erfolgreichen ämterübergreifenden Zusammenarbeit für innen- als auch aussenpolitische Belange im Bereich der Migrationspolitik“"5.

4 PT Balkan, ILR, Strategie Balkan 2003-2006 : Rückkehrhilfeprogramm Balkan für vulnerable Personen (Bosnien und Herzegowina, Serbien und Montenegro, Kosovo): Schlussbericht 2007 (Berichtsperiode 1. Juli 2003 bis 31. Dezember 2006), Bern, BFM; DEZA; IOM, 2007.

5 Eidgenössische Finanzkontrolle (EFK), Bundesamt für Migration, Bereich Rückkehrförderung, EFK Bericht, Nr. 1.6196.420.00145.02, Bern, EFK, 20. Dezember 2006. 


\section{Bilanz der Strukturhilfekomponente am Beispiel des Balkans}

Das „Rückkehrhilfeprogramm Balkan für vulnerable Personen (2003-2006)“ beinhaltete eine vom BFM finanzierte und von der DEZA umgesetzte Strukturhilfekomponente in Höhe von 29 Millionen Franken. Die Umsetzung der Projekte wurde von der Humanitären Hilfe und der Ostzusammenarbeit der DEZA geleitet. Durchführungspartner der DEZA waren lokale Regierungs- und Nichtregierungsorganisationen, internationale Organisationen (IOM, UNHCR) sowie Schweizer Nichtregierungsorganisationen. Insgesamt wurden 49 Projekte von der ILR zur Unterstützung bewilligt.

Die damals durchgeführten Projekte haben bedeutende Beiträge zur Stabilisierung der sozialen und wirtschaftlichen Verhältnisse in den genannten Ländern geleistet. Zusätzlich zur individuellen Unterstützung beschlossen das BFM und die DEZA im Jahr 2003, in ausgewählten Einzelfällen finanzielle Unterstützung für so genannte Ministrukturhilfeprojekte bereitzustellen, welche die lokalen Strukturen in den Rückkehrorten stärken und den Rückkehrern die Reintegration in die lokale Gesellschaft erleichtern sollten. Insgesamt wurden bis Ende 2006 neun vom BFM finanzierte Projekte durch die IOM unter der Leitung der DEZA erfolgreich umgesetzt. In praktisch allen Fällen beteiligten sich die Empfängergemeinden entweder finanziell oder durch Installationsarbeiten an den Projekten. Die gesamthafte Auswertung der Kleinstrukturhilfeaktionen hat jedoch ergeben, dass das Verhältnis von Bearbeitungsaufwand und vorhandenen Finanzmitteln sowie die Nachhaltigkeit von derart punktuellen Aktionen ungünstig sind. Im Rahmen der ILR-Folgestrategie Migrationspartnerschaften Westbalkan 2007-2009 ist deshalb keine Fortsetzung der Ministrukturhilfe geplant.

Die Vernetzung innerhalb des PT Balkan, der Informationsfluss sowie die Unterstützung durch die Kooperationsbüros der DEZA vor Ort haben Modellcharakter für die Zusammenarbeit zwischen BFM und DEZA im Rahmen der ILR in anderen Regionen. Mit dem verstärkten Engagement des BFM in Regionen wie der ehemaligen Sowjetunion oder in Afrika wird auch die Zusammenarbeit mit den für diese Regionen zuständigen Abteilungen in der DEZA gestärkt.

\section{Finanzen}

Mit dem Rückgang der Anzahl freiwillig zurückkehrender Personen seit den grossen Balkanprogrammen wurden auch das Budget der Rückkehrhilfe und somit die effektiven Ausgaben nach unten angepasst.

Auch nahmen die Ausgaben für Strukturhilfe aus dem Verpflichtungskredit kontinuierlich ab und sind heute auf einem konstanten Niveau. Andere Länder und Regionen, wie zum Beispiel Länder der ehemaligen Sowjetunion, Westafrika sowie die Maghrebländer als Migrationstransitrouten, gewinnen an Bedeutung, was zu einer vermehrten Umsetzung von Strukturhilfeprojekten und Projekten zur Prävention irregulärer Migration in diesen Regionen führt (siehe Grafik 2). 
Grafik 2: Ausgaben der Rückkehrhilfe, 2000-2009 (in Millionen Franken)

in Mio. CHF

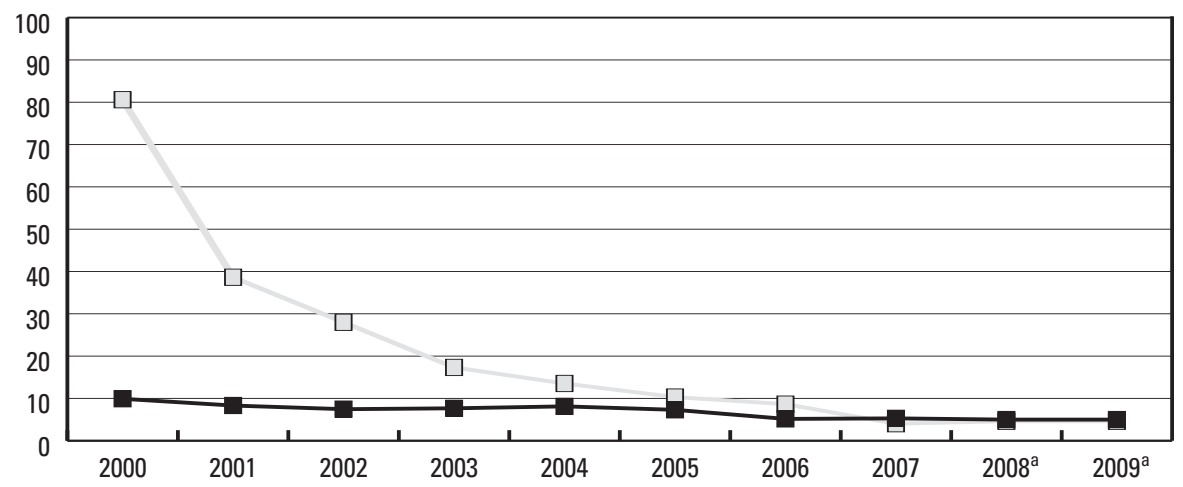

Kredit Rückkehrhilfe (RKH) allgemein ${ }^{\mathrm{b}} \square$ Verpflichtungskreditc ${ }^{\mathrm{c}}, 235$ Mio.

\section{Angaben zu Grafik 2}

\begin{tabular}{lcrr}
\hline & Kredit RKH allgemein & Verpflichtungskredit & Total \\
\hline 2000 & 9911832 & 80600000 & 90511832 \\
\hline 2001 & 8320528 & 38634339 & 46954867 \\
\hline 2002 & 7463968 & 27989425 & 35453393 \\
\hline 2003 & 7664740 & 17326355 & 24991095 \\
\hline 2004 & 8121000 & 13530994 & 21651994 \\
\hline 2006 & 7300000 & 10314450 & 17614450 \\
\hline 2007 & 5153838 & 8644634 & 13798472 \\
\hline $2008^{\text {a }}$ & 5290522 & 4027433 & 9317955 \\
\hline $2009^{a}$ & 5000000 & 4600000 & 9600000 \\
\hline
\end{tabular}

Quelle: Sektion Rückkehrhilfe, BFM, 2008.

a Die Zahlen für die Jahre 2008 und 2009 sind provisorische Angaben.

${ }^{\mathrm{b}}$ Kredit RKH allgemein = Rückkehrberatung und individuelle Rückkehrhilfe.

${ }^{c}$ Verpflichtungskredit = Länderprogramme und Strukturhilfe.

\section{Zukunftsperspektiven}

Das Rückkehrhilfekonzept versteht unter „Rückkehrhilfe“ die Gesamtheit der Massnahmen, die bei asylsuchenden Personen die Bereitschaft zur freiwilligen Ausreise fördern. Die Rückkehrhilfe stellt die vorteilhafte Alternative zur unfreiwilligen Rückkehr dar; auch bleibt sie die einzige Option, wenn diese nicht durchführbar ist. Die Rückkehrhilfe und die Durchführung von Länderprogrammen und Strukturhilfeprojekten tragen zu einer stärkeren Akzeptanz von Seiten der Behörden in den Herkunftsländern bei und sind ein positives Element im Migrationsdialog. Die zurückkehrenden Personen werden bei der Reintegration im Heimatland unterstützt, und es wird versucht, einer weiteren Migration vorzubeugen. Somit ist die Rückkehrhilfe ein wichtiger Pfeiler der Migrationspolitik.

Erstes Ziel und Aufgabe der Rückkehrhilfe des Bundes ist und bleibt es, Personen aus dem Asyl- und teilweise aus dem Ausländerbereich, dazu zu bewegen, die Schweiz freiwillig oder selbständig zu verlassen. Unter „freiwilliger Ausreise“ ist nach schweizerischer Praxis eine Rückkehr zu verstehen, welche ohne 
entsprechende Rechtspflicht erfolgt, da der Ausländer im Besitze eines gültigen Aufenthaltsstatus ist. Als „selbständig“ ist die Ausreise dann zu bezeichnen, wenn das Aufenthaltsrecht des Ausländers in der Schweiz erloschen ist und er innerhalb der behördlich angeordneten Frist und ohne polizeiliche Zwangsmassnahmen das Land verlässt.

Nach den Anpassungen von Umfang und Höhe der individuellen Rückkehrhilfe an die Leistungen in den Länderprogrammen in den letzten Jahren wurde mit Inkrafttreten des neuen Asyl- und Ausländergesetzes zu Beginn des Jahres 2008 die individuelle Rückkehrhilfe auf Verordnungsstufe mit Beträgen festgehalten. Einerseits hat sich die individuelle Rückkehrhilfe im Rahmen dieser Beträge bewährt. Andererseits ist die Rückkehrhilfe an einen Punkt gelangt, an dem mit zusätzlichen Leistungen die Gefahr eines so genannten „Pull-Faktors“6 in die Schweiz eintreten könnte, was zu vermeiden ist. Derartige Erfahrungen wurden im Jahr 2007 gemacht, als eine grosse Anzahl Asylgesuche von Personen aus Rumänien in der Schweiz eingereicht wurden; dies unter anderem mit der Begründung der schwierigen wirtschaftlichen und sozialen Lebensbedingungen im Heimatland und mit der Bemerkung der Betreffenden, sie hätten von besonders vorteilhaften Lebensbedingungen in der Schweiz und von der Gewährung von Rückkehrhilfe gehört. Das BFM entschied deshalb am 7. Mai 2007, dass Personen aus EU-Ländern keinen Anspruch mehr auf Rückkehrhilfe haben. Es gibt derzeit keine Anhaltspunkte, dass die Rückkehrhilfe für Personen aus bestimmten Ländern einen Pull-Faktor bewirkt. Anhand der Asylstatistiken könnte das BFM solche Tendenzen rasch feststellen und entsprechende Massnahmen ergreifen.

Mit dem Pilotprojekt AuG werden erste Erfahrungen an Rückkehrhilfe für Personen im Ausländerbereich gesammelt. Ob das Instrument der Rückkehrhilfe auf weitere Zielgruppen aus dem Ausländerbereich ausgebaut wird, hängt von diesen Erfahrungen und weiteren Entwicklungen ab.

Das Kerngeschäft der Rückkehrhilfe, nämlich die Organisation jeder einzelnen freiwilligen und selbstständigen Rückkehr, bleibt grundlegend. Daneben gehen die Entwicklungen aber auch weg von den langfristigen umfangreichen Länderprogrammen mit Rückkehrhilfe- und Strukturhilfekomponente. Mit dem Ausbau der Leistungen der individuellen Rückkehrhilfe ist die Umsetzung eines Länderprogramms nur noch in speziellen Konstellationen vertretbar. Dies dann, wenn zum Beispiel aufgrund der Situation vor Ort oder einer grösseren Anzahl vulnerabler Rückkehrender eine umfassendere Betreuung oder höhere Leistungen notwendig sind, welche die individuelle Rückkehrhilfe nicht abdecken kann.

Das Angebot von Strukturhilfe und von Projekten zur Prävention irregulärer Migration gilt weiterhin für alle Länder, für deren Staatsangehörige das BFM Rückkehrhilfe leistet. Derartige Projekte sollen auch in Zukunft in erster Linie der Bevölkerung vor Ort zugute kommen. Das Angebot von Strukturhilfe- und PiM-Projekten orientiert sich sowohl an den migrationspolitischen Entwicklungen als auch an migrationspolitischen Interessen des BFM.

6 Bezieht sich auf das sog. Push- und Pull-Faktoren-Modell der Migrationsforschung. Push-Faktoren sind im Herkunftsland vorherrschende Bedingungen, die zur Migration zwingen (Wirtschaftskrisen, Verfolgung, Kriege, etc). Pull-Faktoren sind Anreize für Migranten im Aufnahmeland (wirtschaftliche Prosperität, politische Stabilität, Demokratie, etc.). (Anmerkung der Redaktion). 
Das Schweizer System der Rückkehrhilfe ist im Vergleich zu anderen Staaten in Westeuropa umfassend ausgebaut. Dies führt unter den zurückgekehrten Personen aus verschiedenen EU-Staaten ins Heimatland $\mathrm{zu}$ nicht einheitlichen Voraussetzungen für eine Reintegration. Innerhalb der Europäischen Union werden unter den Staaten in unregelmässigen Abständen Informationen ausgetauscht. Diese werden jedoch nicht analytisch erfasst und eine eigentliche Koordination findet nicht statt. Im Rahmen der migrationspolitischen Zusammenarbeit europäischer Staaten wäre der Austausch im Bereich der Rückkehrhilfe sowie eine Annäherung der verschiedenen Rückkehrhilfeinstrumente und -leistungen wünschenswert.

Fazit

Nicht allein die Anzahl der freiwillig zurückkehrenden Personen ist ein Indiz für eine erfolgreiche Rückkehrhilfe. Vielmehr tragen die verschiedenen Instrumente der Rückkehrhilfe zu einer umfassenden Rückkehrstrategie bei, welche die freiwillige, pflichtgemässe und zwangsweise Rückkehr verlässlich und kooperativ mit dem Herkunftsstaat durchzusetzen versucht. Dies mit dem Ziel, mittel- und langfristig einen Beitrag zur verbesserten migrationspolitischen Zusammenarbeit mit den betreffenden Herkunftsstaaten zu leisten und eine positive Alternative zur unfreiwilligen Rückkehr zu schaffen.

So leistet die Rückkehrhilfe des Bundes zusammen mit ihren Partnern in der Interdepartementalen Leitungsgruppe Rückkehrhilfe (ILR) gezielt Beiträge an rückkehrrelevante, übergeordnete migrations- und aussenpolitische Interessen der Schweiz, wie dies mit dem „Beitrag der ILR zu den Migrationspartnerschaften Schweiz-Westbalkan 2007-2009“ bereits eingeleitet worden ist.

\section{Bibliographie}

Eidgenössische Finanzkontrolle (EFK), Bundesamt für Migration, Bereich Rückkehrförderung, EFK Bericht, Nr. 1.6196.420.00145.02, Bern, EFK, 20. Dezember 2006.

Interdepartementale Leitungsgruppe Rückkehrhilfe (ILR), Beitrag der ILR zu Migrationspartnerschaften Schweiz-Westbalkan: Strategiepapier 2007-2009 Kosovo, Bosnien und Herzegowina, Serbien, Bern, Bundesamt für Migration (BFM); Direktion für Entwicklung und Zusammenarbeit (DEZA), 2007.

Interdepartementale Leitungsgruppe Rückkehrhilfe (ILR), Mandat, Bern, Bundesamt für Migration (BFM); Direktion für Entwicklung und Zusammenarbeit (DEZA), 2005.

PT Balkan, Interdepartementale Leitungsgruppe Rückkehrhilfe (ILR), Strategie Balkan 2003-2006: Rückkehrhilfeprogramm Balkan für vulnerable Personen (Bosnien und Herzegowina, Serbien und Montenegro, Kosovo): Schlussbericht 2007 (Berichtsperiode 1. Juli 2003 bis 31. Dezember 2006), Bern, Bundesamt für Migration (BFM); Direktion für Entwicklung und Zusammenarbeit (DEZA); Internationale Organisation für Migration (IOM), 2007.

Sektion Rückkehrhilfe BFM, Grundlagenpapier zuhanden der ILR 2008 : Operationelle Instrumente der Rückkehrhilfe, Bern, Bundesamt für Migration (BFM), 2008. 